\title{
Patient Centricity and the Ethics of Glaucoma Care
}

\author{
Shibal Bhartiya
}

\begin{abstract}
The ultimate goal of glaucoma therapy, as of any other therapeutic intervention, is to achieve superior clinical outcomes, patient satisfaction, and patient adherence to treatment. In a chronic asymptomatic disease, such as, glaucoma, where diagnostic and therapeutic algorithms may have multiple acceptable treatment arms, patient centricity becomes increasingly important. Shared decision-making, patient participation, quality of life $(\mathrm{QoL})$ concerns, and risk-benefit analyzes further complicate this decision-making. In addition, the ethics of research in glaucoma and also that of glaucoma screening may often be in conflict with the ethics of patient care. This article aims to highlight the ethical dilemmas that confound decision-making in current glaucoma practice, and the doctors' fiduciary duties to the patient.

Keywords: Ethics, Glaucoma practice, Patient centricity, Quality of life, Shared decision-making.

Journal of Current Glaucoma Practice (2020): 10.5005/jp-journals-10078-1281
\end{abstract}

"Nothing is true, all is permitted".

-Frederick Nietzsche

\section{INTRODUCTION}

Glaucoma remains the most important cause of irreversible blindness globally, and its management remains a challenge. It is a chronic, progressive, and largely asymptomatic disease, often made symptomatic due to the treatment provided. In a Nietzschean parable, no "one" established management option is always true, yet almost all are permitted, for each patient. Moreover, the interaction between disease characteristics, patient demographics and preferences, compliance, economics, and institutional/individual capabilities complicates the decision-making algorithm. ${ }^{1-5}$ If that was not enough, the rapid advances in minimally invasive glaucoma surgery (MIGS) and the diversity of its applications make clinical choices even more difficult, particularly as most of these treatment options lack strong level 1 evidence and unified consensus.

The elusive holy grail of glaucoma care, therefore, is the perfect trifecta of superior clinical outcomes, patient satisfaction, and patient adherence to treatment.

\section{Patient Centricity and Value-based Customization of Treatment Plans}

While the standard of care is largely established for glaucoma type and severity, most therapeutic algorithms have multiple acceptable treatment arms. Additionally, individual deviations from the care plans are completely acceptable depending on patient characteristics, especially comorbidities.

The latter is an important consideration, as glaucoma often affects the elderly, the presence of comorbidities is significant. In glaucoma patients, the incidence of hypertension has been reported from 29 to $48 \% ; 16$ to $35 \%$ had coronary artery disease, while 9 to $17 \%$ had diabetes, and 9 to $17 \%$ had thyroid disease. ${ }^{2,3}$ The incidence of asthma, depression, and congestive heart failure was reported to be $7 \%, 8 \%$, and $12 \%$, respectively. ${ }^{3}$ Therefore, the individualized patient-centric choice must consider factors seemingly unrelated to the incident disease itself, since these comorbidities affect quality of life (QoL), life expectancy, as well as the choice of medications.
Department of Ophthalmology, Glaucoma Services, Fortis Memorial Research Institute, Gurugram, Haryana, India

Corresponding Author: Shibal Bhartiya, Department of Ophthalmology, Glaucoma Services, Fortis Memorial Research Institute, Gurugram, Haryana, India, Phone: +91 9818700269, e-mail: shibalbhartiya@gmail.com

How to cite this article: Bhartiya S. Patient Centricity and the Ethics of Glaucoma Care. J Curr Glaucoma Pract 2020;14(2):68-71.

Source of support: Nil

Conflict of interest: None

Patient characteristics include the severity of disease, as well as demographics. QoL preservation, by way of disability limitation and prevention of blindness, in the long-term is to be weighed against the immediate and ongoing impact on the same. QoL costs of any intervention, thus, are central to decision-making, since they impact patient adherence. Each individual patient will present with a unique set of concerns for the same diagnosis. That said, usually, for the same severity of disease, the treatment must be more aggressive for a younger patient, but must also take into account the QoL costs of the treatment. Since the efficacy of most glaucoma medical treatments is not easily quantifiable by objective measures, patient-centric approach empowers the patient to take ownership, thus improving patient adherence to treatment, and consequently, clinical outcomes. ${ }^{4,5}$

\section{Shared Decision-making}

Only when we acknowledge, and incorporate our patients' values, goals, and concerns, into our treatment plans, do we move away from the patriarchal medical care, to empathetic patient care. Sadly, for most practices, this also means taking into account financial realities and social constraints.

Patient centricity emphasizes not only customization of treatment to the patients need but also his or her active participation in therapeutic decision-making. Patients' values, priorities and perceptions of QoL, and functional outcome also affect the decision-making algorithm. ${ }^{1,5-7}$ This is especially true for glaucoma, since very rarely is there one best treatment option,

(c) The Author(s). 2020 Open Access This article is distributed under the terms of the Creative Commons Attribution 4.0 International License (https://creativecommons. org/licenses/by-nc/4.0/), which permits unrestricted use, distribution, and non-commercial reproduction in any medium, provided you give appropriate credit to the original author(s) and the source, provide a link to the Creative Commons license, and indicate if changes were made. The Creative Commons Public Domain Dedication waiver (http://creativecommons.org/publicdomain/zero/1.0/) applies to the data made available in this article, unless otherwise stated. 
and all therapy invariably comes with a trade-off between risks and benefits. Several patients may opt against lifelong medication, while others will try to avoid surgery even in advanced disease. Yet, another consideration is guiding patients' choice: Helping them make an informed, intelligent decision, consistent with their life priorities.

Sometimes institutional or individual abilities to offer certain therapeutic modalities may be limited. Most of the developing world does not have access to MIGS or lasers, many surgeons are not trained in tubes/MIGS/non-penetrating glaucoma surgeries. For many glaucoma practitioners, the affordability and availability of prostaglandin analogs, let alone the newer Rho kinase inhibitors (ROCK inhibitors), is still an important consideration.

Before any conversation about treatment options, the patient must be explained the diagnosis and its implications, prognosis, and the therapeutic options available.

While Charles describes the conversation in head and neck cancers, ${ }^{8}$ the basic tenets of communication remain the same.

- Sharing treatment benefits and risks.

- Patient sharing of treatment goals.

- Discussion of treatment options.

- A formal agreement of an established plan.

Often social perceptions and constraints, as well as financial considerations will guide these choices for the patient. When the priorities of the patient and caregiver diverge, especially with finite healthcare funds, the need for the optimal therapeutic decision to maximize clinical outcomes becomes paramount. Also, in case the recommended treatment plan is different from what the patient prefers, a discussion that results in a consensus becomes mandatory.

\section{Ethics of Glaucoma Care}

Clinical ethics requires that general principles and concepts must be applied with discretion and intelligence to varied clinical scenarios. In this, it is analogous to the clinical practice of glaucoma. The guidelines that govern clinical ethics are also as nebulous as the guidelines for glaucoma practice: A fine, often indiscernible, line divides what is best for the patient, what is perceived to be the best, and what is the best possible in a given clinical situation. ${ }^{9}$

Of course, there are areas where the guidelines are clear. Fee splitting, excessive charges to patients, and deficient consent or confidentiality are not only unethical but also not permitted by policy and law. This actually questions the integrity of the physician, and the ability of the doctors to regulate their conduct. ${ }^{9-15}$

Any potential conflict of interest, financial or otherwise, is as damaging in intent. If the welfare of the patient is not central to the intervention, and the patient receives the optimal care, it would have happened despite the potential for impaired objectivity. This not only exposes the patient to a potential risk but also corrodes the very basis of a doctor-patient relationship. 9,15

For almost every ethical dilemma, the standards are guided by the doctors' fiduciary duty to the patient: Trust is central and critical. Glaucoma surgeons, indeed, like all their other medical colleagues, must embrace trust as the matrix of healthcare. Beauchamp and Childress have characterized the principle perspective of biomedical ethics. ${ }^{16}$

The four principles, believed to be of equal importance are respect for patient autonomy; beneficence; non-maleficence; and justice.
- Patient autonomy: This is the most commonly used principle for guiding the ethical dilemma in the glaucoma clinic. Patients often seem to not take their glaucoma seriously, and often avoid medication or surgery. These decisions are often irrational, and have potential to harm their vision. However, given the largely asymptomatic nature of the disease their behavior is easily explained. It is then the physicians' fiduciary duty to help the patient make the right choice. In patients with secondary glaucomas or angle closure glaucoma where the rate of progression is faster than in open angle glaucomas, the doctor may gently curtail patient autonomy and work for preservation of vision instead.

- Beneficence: It is incumbent on the physician to always act in the best interest of the patient, throughout the patient journey from diagnosis to treatment. Indeed, it is the essence of the discipline of medicine itself, but is often in conflict with the principle of patient autonomy, which brings into play patient preferences, values, and perceptions. Once again, disagreements about what constitutes the patient's good and who should decide that, often is a trap for falling into the patriarchal mindset of doctor knows best.

- Non-maleficence: Primum Non Nocere, first do no harm, is central to the Hippocratic Oath. This principle is important in situations where the risks of glaucoma surgery have to be weighed against the potential complications of the same. Also, the QoL costs of each intervention may also be carefully weighed, especially since almost any glaucoma treatment potentially can make a hitherto comfortable patient symptomatic. Provocative tests, though performed very rarely in current glaucoma practice, which may precipitate an angle closure also fall in the purview of this dictum.

Quality of life assessments like time trade-off, standard gamble, and utility values may help guide the physicians decision-making in case of this ethical dilemma. Additionally, a better understanding of patient-reported QoL and patient apprehensions can improve patient-physician interaction and treatment adherence, thereby resulting in better long-term visual prognosis.

- Justice: Justice has two equally important principles: Equitability and distributive justice. Equitability means that all patients of glaucoma with similar severity must be provided the same quality of medical care regardless of non-medical factors, such as, wealth or insurance cover and social capital. Distributive justice, on the contrary, recognizes the fact that healthcare resources are limited, and must be distributed fairly among all patients, determined only by their healthcare needs. The concept of justice has come into sharp focus as we discuss healthcare access and universal insurance across geopolitical boundaries. This is especially true in situations where patients of advanced glaucoma are denied access to effective surgical interventions, while those with early disease may receive costly surgical procedures, often multiple, based solely on insurance reimbursements and ability to pay. Similarly, many practices still use timolol as the first-line drug for medical management of glaucoma, despite the proven superiority of prostaglandin analogs, simply because the former is cheaper.

Also, in a systematic violation of principles of distributive justice, there have been reports that many glaucoma patients have progressed to blindness during the coronavirus disease-2019 (COVID-19) pandemic, as this subgroup of asymptomatic patients 
were denied care, because the crisis standards of public health took precedence over the needs of the individual patient. ${ }^{18}$ This population-based resource allocation following risk stratification, will continue to haunt glaucoma surgeons in the years to come, as we struggle to come to terms with the post COVID-19 world. ${ }^{19}$

\section{Ethics of Glaucoma Research}

It is also imperative to distinguish between the ethics of clinical practice and that of glaucoma research. ${ }^{9}$ Foremost is the fact that in a clinical setting, the primary aim is to ensure the vision-related QoL of the patient. When enrolled in clinical research, on the contrary, the individual patient actually gives consent to be part of an evaluation, which may or not benefit them (e.g., for those on the placebo arm of a randomized control trial); or where the patient may actually not know whether they are receiving any treatment at all for the disease that they are diagnosed with (blinded trials). For those on placebo arms, the disease may continue to progress while they are on treatment, despite them adhering to their doctors' instructions to the letter. Crossover trials address this ethical dilemma, but the loss of time on the placebo arm may not be entirely remain justifiable. Moreover, the intended treatment may cause side effects hitherto unknown, in case the patient is prescribed a new drug, and the patient may suffer from the consequences over time. Since participating in research may not benefit the patient, and may even be harmful, the ethical obligations for the doctor are manifold. The most important is informed consent, where the patient understands that their enrolment in a clinical trial may not be to their benefit, but will help the public at large. They must also understand completely the implications of placebo therapy, and a full disclosure of any anticipated adverse is mandatory. Needless to say, there should be no coercion for enrolment into clinical trials. In addition, the patient must also know that withdrawal of consent to participate in the clinical trial would in no way impact his or her treatment, or relationship with the doctor.

\section{Ethics of Glaucoma Screening}

The ethical dilemma of glaucoma screening protocols is obvious. ${ }^{1}$ Even in the best hands, glaucoma diagnosis is inexact and timeconsuming. More than anything else, glaucoma management, and very often the diagnosis as well, requires long-term follow-up. A generic one-time screening program, thus, may result in a high number of both, false-negatives, and false-positives. Given that population-based glaucoma screening is not cost-effective, or feasible logistically, a screening of high-risk groups may be carried out. But given that low risk is not synonymous with any risk, this again presents a unique ethical dilemma.

This is even more pronounced in cases of ocular hypertension, since not all patients progress to visual field damage, let alone visual disability. In those that do, the damage caused is irreversible, so for this subgroup, to wait for long-term sequential follow-up before instituting therapy may just not be the best choice.

The available treatment algorithms are imprecise, and require constant modification, which is not a possibility following a screening alone. Also, fear of blindness, cost of therapy, and loss of working days mean that the QoL of the patient is adversely affected by the diagnosis itself. In case the patient is unable to afford therapy or further diagnostic testing, a glaucoma screening is actually to the patients' detriment. In such a case, is it better for the doctor decide to not inform the patient of diagnosis and need for treatment, or to not offer screening services at all?

\section{Conclusion: A Relationship of Inviolate TRUST}

Current glaucoma practice can no longer afford to be patriarchal, and needs to break away from the traditional hierarchical structure, where the doctor makes all decisions. Shared decision-making and patient centricity are the cornerstones of glaucoma care, and constitute good clinical practice, optimizing clinical outcomes.

Increasing patient-centeredness and participation in treatment plans increases patient satisfaction and results in greater tolerance of uncertainty. While this is important in overall glaucoma management, it is invaluable, especially in the context of glaucoma surgeries. Social justice, inequalities, and compromised physical and mental health are also important considerations while taking care of a glaucoma patient.

While the ethics of patient centricity is inviolate, a conflict between the four ethical principles may complicate clinical decision-making. Current practice of glaucoma is peppered with potential ethical challenges and requires the treating ophthalmologist to forever think of the patients' best interests. An open, equal, and frequent communication, together with tailoring the management protocol to the individuals' needs is perhaps the only way to negotiate this ethical quagmire.

\section{References}

1. Clinical Decision Making in Glaucoma Bhartiya S 1st ed., New Delhi: Jaypee Brothers Medical Publishers; 2018.

2. Canadian Glaucoma Study Group. Canadian glaucoma study: study design, baseline characteristics and preliminary analyses. Can J Ophthalmol 2006;41(5):566-575. DOI: 10.1016/S0008-4182(06) 80025-6.

3. Gottfredsdottir M, Allingham R, Shields M. Physicians' guide to interactions between glaucoma and systemic medications. J Glaucoma 1997;6(6):377-383. DOI: 10.1097/00061198-19971200000007.

4. Chi JJ. Reflections on shared decision making. Otolaryngol Head Neck Surg 2018;159(5):809-810. DOI: 10.1177/0194599818792212.

5. Elwyn G, Dehlendorf C, Epstein RM, et al. Shared decision making and motivational interviewing: achieving patient centered care across the spectrum of health care problems. Ann Fam Med 2014;12(3):270-275. DOI: 10.1370/afm.1615.

6. Politi MC, Street Jr RL. The importance of communication in collaborative decision making: facilitating shared mind and the management of uncertainty. J Eval Clin Pract 2011;17(4):570-584. DOI: 10.1111/j.1365-2753.2010.01549.x.

7. Pollard S, Bansback N, Bryan S. Physician attitudes toward shared decision making: a systematic review. Patient Educ Couns 2015;98(9):1046-1057. DOI: 10.1016/j.pec.2015. 05.004.

8. Charles CA. Shared treatment decision making: what does it mean to physicians? J Clin Oncol 2003;21(5):932-936. DOI: 10.1200/ JCO.2003.05.057.

9. Taylor RM. Ethical principles and concepts in medicine. Handb Clin Neurol 2013;118:1-9.

10. International Academy of Ophthalmology.. Code of ophthalmological ethics. Trans Ophthalmol Soc UK 1978;98:514-520.

11. American Academy of Ophthalmology. Code of Ethics. http:// www. aao.org/about/ethics/code_ethics.cfmpreamble. Accessed January 25, 2020. 
12. Bettman JW. Ethics and the American Academy of Ophthalmology in historical perspective. Ophthalmology 1996;103:S29-S39. DOI: 10.1016/S0161-6420(96)30762-8.

13. Dixon-Woods M, Yeung K, Bosk CL. Why is U.K. Medicine no longer a self-regulating profession? the role of scandals involving "bad apple" doctors. Soc Sci Med 2011;73(10):1452-1459. DOI: 10.1016/j. socscimed.2011.08.031.

14. Bettman JW. The development of the code of ethics for the American Academy of Ophthalmology. Surv Ophthalmol 2000;44(4):357-359. DOI: 10.1016/S0039-6257(99)00118-6.

15. Levin AV, Ganesh A, Al-Busaidi A. Fee splitting in ophthalmology. Can J Ophthalmol 2011;46(1):21-27. DOI: 10.3129/i10-097.
16. Beauchamp TL, Childress JF. Principles of Biomedical Ethics. 6th ed. New York: Oxford University Press; 2008.

17. Quaranta L, Riva I, Gerardi C, et al. Quality of life in glaucoma: A review of the literature. Adv Ther 2016;33(6):959-981. DOI: 10.1007/ s12325-016-0333-6.

18. https://www.telegraph.co.uk/news/2020/01/09/patients-goingblind-long-nhs-delays-investigators-warn/ Accessed 25 June, 2020.

19. Jayaram H, Strouthidis NG, Gazzard G. The COVID-19 pandemic will redefine the future delivery of glaucoma care. Eye 2020;34(7):12031205. DOI: 10.1038/s41433-020-0958-1. 